
\title{
25 Research Soure \\ Potential Influence of Factors For Genu Valgus With Hereditary Multiple Exostoses
}

\author{
Ya Liu
}

Soochow University Affiliated Children's Hospital

Jianfeng Fang

Soochow University Affiliated Children's Hospital

Yao Liu

Soochow University Affiliated Children's Hospital

Zheng Zhang

Soochow University Affiliated Children's Hospital

Xiaodong Wang

Soochow University Affiliated Children's Hospital

Zhixiong Guo ( $\nabla$ zhizhe4382116@sina.cn )

Soochow University Affiliated Children's Hospital https://orcid.org/0000-0001-5654-2421

Fuyong Zhang

Soochow University Affiliated Children's Hospital

Keywords:

Posted Date: May 13th, 2021

DOl: https://doi.org/10.21203/rs.3.rs-491364/v1

License: (c) (1) This work is licensed under a Creative Commons Attribution 4.0 International License. Read Full License

Version of Record: A version of this preprint was published at Journal of Pediatric Orthopaedics B on February 14th, 2022. See the published version at https://doi.org/10.1097/BPB.0000000000000955. 


\section{Abstract}

The authors have requested that this preprint be removed from Research Square. 